\title{
Spectral features based tea garden extraction from digital orthophoto maps
}

\author{
Akhtar Jamil, ${ }^{\text {a } B u l e n t ~ B a y r a m, ~}{ }^{\text {a }}$ Turgay Kucuk, ${ }^{b}$ Dursun Zafer Seker ${ }^{\mathrm{c}}$ \\ ${ }^{a}$ Yildiz Technical University, Department of Geomatics Engineering, Istanbul, Turkey; akh.jam@yildiz.edu.tr, \\ bbyram@yildiz.edu.tr \\ ${ }^{b}$ General Director, EMI Information Technologies Inc., turgay@emigrup.com.tr \\ ${ }^{c}$ Istanbul Technical University, Civil Engineering Faculty, Department of Geomatics Engineering; seker@itu.edu.tr
}

\begin{abstract}
The advancements in the photogrammetry and remote sensing technologies has made it possible to extract useful tangible information from data which plays a pivotal role in various application such as management and monitoring of forests and agricultural lands etc. This study aimed to evaluate the effectiveness of spectral signatures for ex-traction of tea gardens from 1:5000 scaled digital orthophoto maps obtained from Rize city in Turkey. First, the normalized difference vegetation index (NDVI) was derived from the input images to suppress the non-vegetation areas. NDVI values less than zero were discarded and the output images was normalized in the range $0-255$. Individual pixels were then mapped into meaningful objects using global region growing technique. The resulting image was filtered and smoothed to reduce the impact of noise. Furthermore, geometrical constraints were applied to re-move small objects (less than 500 pixels) followed by morphological opening operator to enhance the results. These objects served as building blocks for further image analysis. Finally, for the classification stage, a range of spectral values were empirically calculated for each band and applied on candidate objects to extract tea gardens. For accuracy assessment, we employed an area based similarity metric by overlapping obtained tea garden boundaries with the manually digitized tea garden boundaries created by experts of photogrammetry. The overall accuracy of the proposed method scored $89 \%$ for tea gardens from 10 sample orthophoto maps. We concluded that exploiting the spectral signatures using object based analysis is an effective technique for extraction of dominant tree species from digital orthophoto maps.
\end{abstract}

Keywords: Tree segmentation, tea garden classification, normalized difference vegetation index, morphological processing, region growing

\section{Introduction}

The advancements in the remote sensing technologies has made it possible to extract useful tangible information which plays a pivotal role in various application such as management and monitoring of forests, crops, agricultural land etc. (Zhou et al. 2016). High resolution multispectral imagery data is widely used for the aforementioned applications. Although multispectral imagery provides detailed information, nevertheless such details can introduce intra-class variations and inter-class similarities there by escalating the complexity of the problem at hand (Paula. et al. 2010; Qin 2014). It requires extraction of highly discriminative features that could distinguish between various tree species.

In recent years, photogrammetry and remote sensing techniques have been exploited for vegetation type classification for assessment of agricultural landscape (Pham, Brabyn, and Ashraf 2016). A variety of sensors are available to obtain high resolution images, with high spectral and spatial resolution: space borne and airborne multi-spectral imagers, Synthetic Aperture Radar (SAR) and airborne Light Detection and Ranging (LiDAR) (Palenichka et al. 2013). The choice of sensor is very important and application specific. For example, to detect large vegetation areas such as tea gardens, a coarse resolution image can be used. On the other hand, if the objective is to detect fine scale objects, such as individual trees, a high spectral resolution imagery data is more useful. Most of the studies have used visible and near infrared bands of the spectrum for tree specifies classification (Dalponte et al. 2013). Additionally, combining heterogeneous data obtained from various sensors is also useful as they provides complementary information about classes of interest (Bandyopadhyay, van Aardt, and Cawse-Nicholson 2013; Colgan et al. 2012; Sugumaran and Voss 2007).

A number of methods employed various indices for extraction of vegetation information from remote sensing data (Pena-Barragana et al. 2004). Traditionally, NDVI has widely been used to distinguish vegetation and nonvegetation areas (Adhikari et al. 2015). In addition, other vegetation indices have been proposed such as excessive green index (Qin 2014), greenness and brightness (Adhikari et al. 2015), ratios between spectral bands (Pena-Barragana et al. 2004) etc. as a measure for vegetation information extraction. These methods exploit spectral reflectance information captured by sensors that characterizes the vegetation.

Generally, the image analysis methods employ either pixels or objects as the basic unit of measurement. Many methods have employed pixel-based methods for image analysis (Deng et al. 2014). However, applying pixelbased methods on high spatial resolution images pose substantial challenges for image segmentation due to 
inter-class spectral variations. On the other side, object based image analysis (OBIA) methods employ objects as basic unit of measurement (Blaschke 2010). OBIA has received considerable attention in the recent era and it has widely been used for remote sensing applications (Colgan et al. 2012; Sugumaran and Voss 2007; Tzotsos and Argialas 2008; Walter 2004). It offers various advantages over traditional pixel based methods, e.g. it reduces the inter-class variations and further information can be derived from objects such as spatial and contextual information. These characteristics make OBIA a strong candidate for remote sensing image analysis.

The objective of this research is to identifying tea gardens from 1:5000 scaled digital orthophoto maps obtained from Rize city in Turkey. The tea trees are small in size and appear in groups which offer a unique texture.. We exploited these characteristics to distinguish it from other types of vegetation. There are two primary objective of the proposed study: 1) to transform the raw pixels into a meaningful object representation using region growing technique. 2) classification of tea gardens from rest of vegetation. Traditional NDVI index was employed to distinguish between vegetation and non-vegetation areas. Then, a regions growing technique was used to transform the pixels into an object-based representation. Some geometrical and morphological techniques were also applied to suppress the non-candidate objects. Finally, a spectral range filter was applied on the candidate objects to obtain the tea gardens. In order to improve further accuracy of the output, an optional manual editing step was also added in the final release of the software. The proposed method was implemented in MATLAB and Emgu CV.

\section{Study Area}

Rize is located in the eastern Black Sea part of Turkey (Coordinates: $41^{\circ} 01^{\prime} 29^{\prime \prime} \mathrm{N} 40^{\circ} 31^{\prime} 20^{\prime \prime} \mathrm{E}$ ). The region is characterized by heavy rain and landscape which has both valleys and steep mountain ranges. The region is naturally more suitable for agriculture and forest and is popular for tea production. The high quality tea is not only distributed across the country but it is also exported to other countries in Europe and Asia. The area in Rize is mostly dense with various vegetation types, including tea and other types of vegetation. Moreover, build areas, roads, and bare land can also be seen in the images. Fig. 1 (a) shows the map of the Rize while (b) shows a sample image of a tea garden.

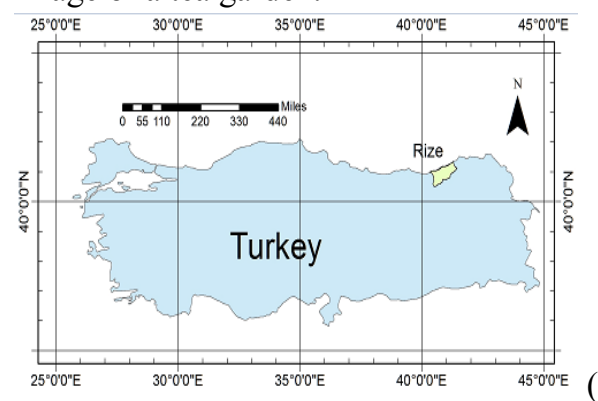

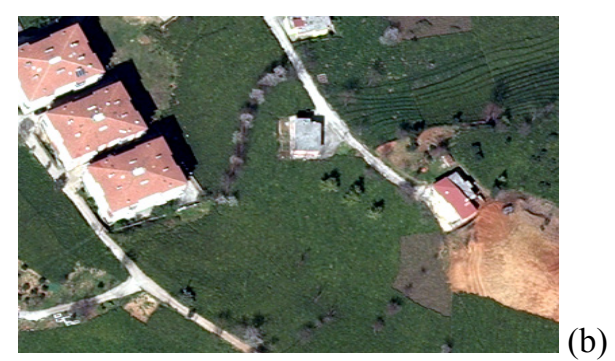

Fig. 1. Study site for the current work. a) Map of Rize city in Turkey. B) A sample image (R, G, B)

\section{Materials and Method}

The aim of this study is to automatically extract the boundaries of tea garden from digital orthophoto maps. The images were preprocessed by applying Gaussian low pass filter to remove noise such as salt and pepper effects. NDVI was then derived from preprocessed images to obtain vegetation areas. Then, region growing technique was employed to transform the raw pixels into an object based representation. Geometrical and morphological operations were applied to remove noisy elements, such as small objects, holes within objects etc. Finally, for classification a range filter was applied to the spectral signature to distinguish tea gardens from rest of vegetation and other types of land cover classes. The main workflow is divided into four sub-tasks (Fig. 2), namely: normalized difference vegetation index calculation, image segmentation, image filtering, and classification. The following sections describe each step in details.

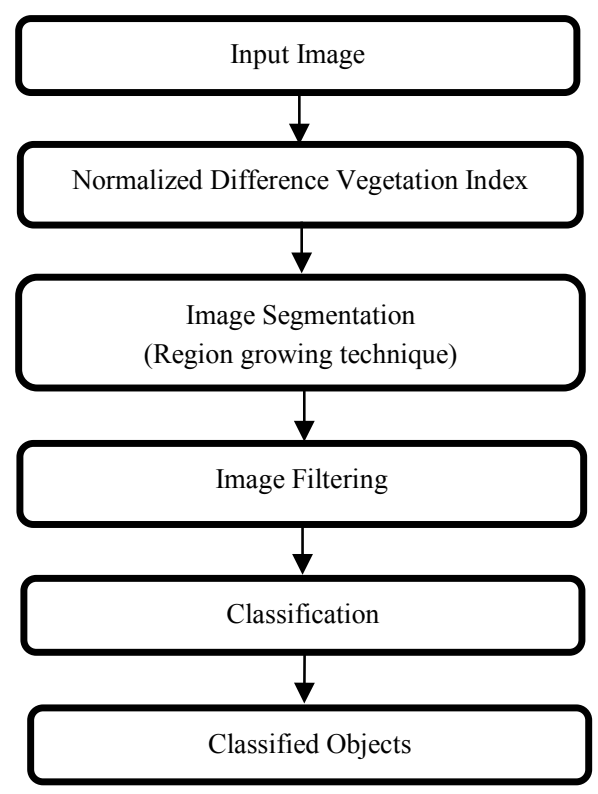

Fig. 2: Workflow of the proposed method for tea garden extraction from digital orthophoto maps

\subsection{Dataset}

The data used for the current study was provided by EMI group, which was obtained on March 03, 2013 using ULTRACAM-X digital aerial camera. The flight was 
made over tea gardens in various regions in Rize city. The data was acquired to make assessments of the area covered by tea gardens in the region. It covered both visible and near infrared parts of the electromagnetic spectrum. The acquired images were then ortho-rectified and scaled to 1:5000 so that direct measurement could be performed. Four spectral bands were used for the current study: red $(\mathrm{R})$, green $(\mathrm{G})$, blue $(\mathrm{B})$ and near infrared (NIR). The characteristics of each band are summarized in Table 1(Alvarez et al. 2010).

\begin{tabular}{ll}
\hline Band Name & Wavelength (nm) \\
\hline Blue & $400-600$ \\
Green & $480-660$ \\
Red & $580-720$ \\
Near Infrared & $620-1000$ \\
\hline
\end{tabular}

Table 1. Summary of band information

\subsection{Normalize Difference Vegetation Index}

Since the images contained collection of heterogeneous objects in addition to tea gardens, such as other types of vegetation and manmade objects (houses, roads, forest etc.), therefore, naturally, the initial step was to distinguish vegetation from non-vegetation areas, and then proceed to distinguish tea gardens from rest of the vegetation. This preprocessing step offered two main advantages, (i) obtaining vegetation areas only (ii) reduction of processing time. Fig. 3 (a) shows the NDVI image obtained from original image while (b) shows the image after thresholding and normalization.

For vegetation area extraction, the normalized difference vegetation index (NDVI) was derived from the input image. NDVI values are obtained by taking the ratio between difference and sum of NIR and R bands of the multispectral image (Jeevalakshmi, Reddy, and Manikiam 2016). Mathematically:

$N D V I=\frac{(N I R-R)}{(N I R+R)}$

Normally, the values of NDVI lie in the range of -1 and +1 . Higher values in the NDVI indicate the presence of high vegetation while lower values indicated less or nonvegetation. In our experiment, we only considered values greater than threshold value 0.2 , which is an indicator for presence of green patches, while values less than the threshold value were discarded (i.e. set to 0 ). The resulting image was normalized to the range $0-255$.
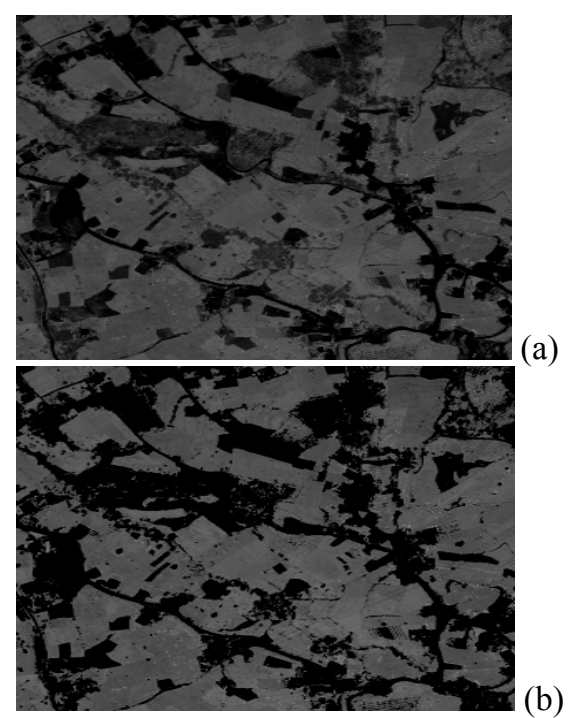

Fig. 3 (a) NDVI image obtained from original image (b) Image after thresholding and normalization.

\subsection{Image Segmentation}

The main objective of image segmentation is to partition the image into a set of disjoint regions which are homogeneous segments based on a similarity metric such as gray level, texture or color. We employed region based image segmentation as it is stable against noise and produces a thin boundary between different regions ( $\mathrm{Li}$ et al. 2015). The algorithm examines each pixel and adds to the regions if the gray intensities are similar to the region. Otherwise, the pixel is discarded and set as background (intensity is set to 0 ). Moreover, we employed a thresholding technique for region growing based on the average gray value for the set of seed points and a user define threshold $(\mathrm{t})$.

We proceed to segment the image by selecting a set of seed points from the NDVI image. The user manually selects these seed points from the region of interest in the image. The gray values for these seed points are obtained and averaged to get their mean value $(\mathrm{m})$. Moreover, a threshold value ( $\mathrm{t}$ ) is also defined by the user which adds an extra constraint so that only those pixels will be added to the region which are within the range of upper and lower constraints obtained from mean and threshold values. To create homogeneous objects, the proposed algorithm then scans the whole image pixel by pixel from right to left and top to bottom and add each pixel to the region if it satisfy

$g(x, y)=\left\{\begin{array}{l}1 \quad \text { if }(m-t) \leq I(x, y) \leq(m+t) \\ 0 \text { otherwise }\end{array}\right.$

Where $\mathrm{g}$ is the filtered image, $\mathrm{I}$ is the NDVI image and $\mathrm{g}(\mathrm{x}, \mathrm{y})$ and $\mathrm{I}(\mathrm{x}, \mathrm{y})$ represents intensities at the location $(\mathrm{x}, \mathrm{y})$ for filtered and NDVI images respectively. Here, $g$ is a binary image where foreground pixels are candidates for vegetation areas while background represents nonvegetation areas. These segments are then used as building blocks for classification described in the following sections. 


\subsection{Image Filtering}

The image segmentation resulted in an object-based representation of the candidate tea garden objects. This representation, however, also contained noisy elements such as small objects and other isolated green patches. Naturally, these noisy objects should be removed first before proceeding to the next steps. To do so, we exploited morphological processing, applied geometrical constraints and hole filling procedures to suppress the non-candidate objects. Fig. 4 shows the results obtained for each step.

Mathematical morphological is a technique for analyzing the geometrical structure of images ( $\mathrm{Gu}$ et al. 2016). The output image is obtained from morphological operation by comparing the corresponding pixels in the input image within a fixed neighborhood. The size and shape of neighborhood is defined by a template called structuring element. We employed morphological opening on the binary image obtained from segmentation step. Morphological opening performs erosion followed by dilation. Dilation tends to add pixels towards the boundaries of objects, while erosion tends to remove pixels along the boundaries. A square structuring element of size $5 \times 5$ was applied on the segmented image. This resulted in removing small gaps between objects and smoothing the object boundaries.

A set of geometrical constraints were then applied to further refine output image. Connected components were calculated and bounding boxes were defined for each object. The geometrical constraints were then applied and the objects were removed with areas less than 500 pixels, width and height of the bounding box less than 200 pixels. This removed small objects which were not candidate tea garden objects.

Finally, small holes were filled within the candidate tea garden objects which were not eliminated by the morphological operation. To achieve this goal, the binary images were complemented and connected component were calculated. Again, geometrical constraints were applied to remove objects whose areas were less than 100 pixels. To keep the candidate tea garden objects in white while background in dark color, the filtered image was complemented.

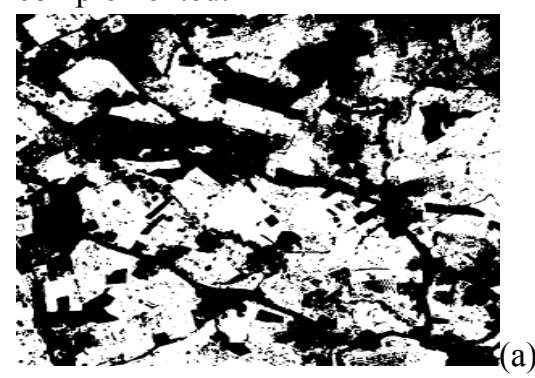

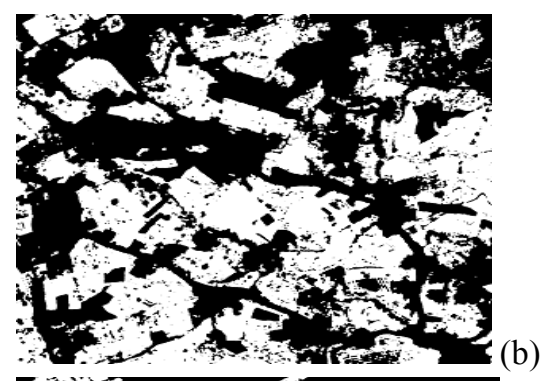

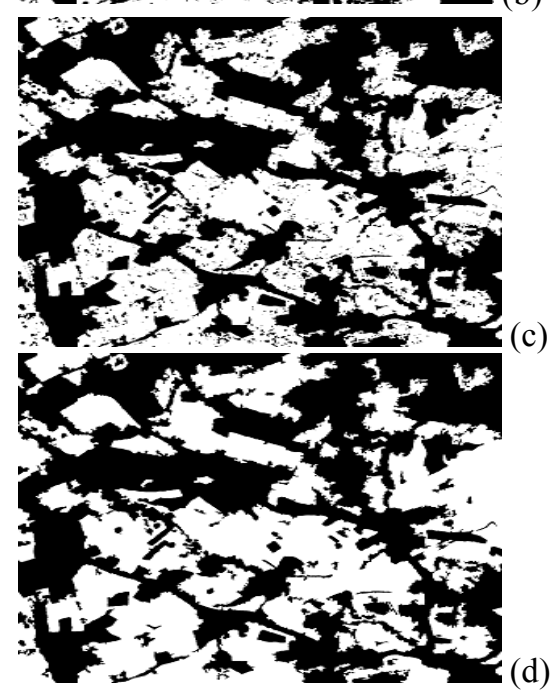

Fig 4. Sample outputs obtained for each intermediate steps. (a) Regions growing image segmentation, (b) Morphological Opening, (c) Area based geometrical constraints applied to remove small regions and (d) Application of hole-filling to remove small holes within objects

\subsection{Classification}

Taking spectral signatures into account, the final classification of objects into tea gardens and rest of land cover classes was obtained. A spectral range was empirically calculated by observing the spectral values for tea gardens. For each candidate object obtained from previous step, we calculated mean spectral values by using spatial contextual information. Then, final range filter was applied to classify the objects into either tea gardens or rest of land cover classes. An object is assigned to tea gardens if its mean spectral value lies in the specified range otherwise, it is discarded assuming it is a non-tea object. Fig. 5 (a) shows the results obtained for classification and (b) shows the boundaries for each tea gardens object.

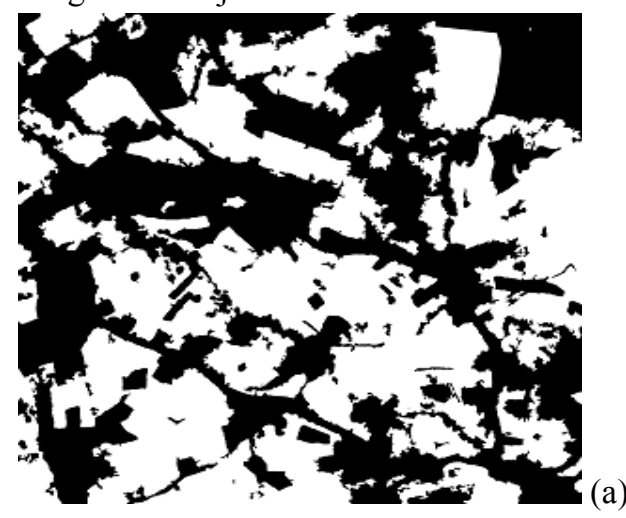




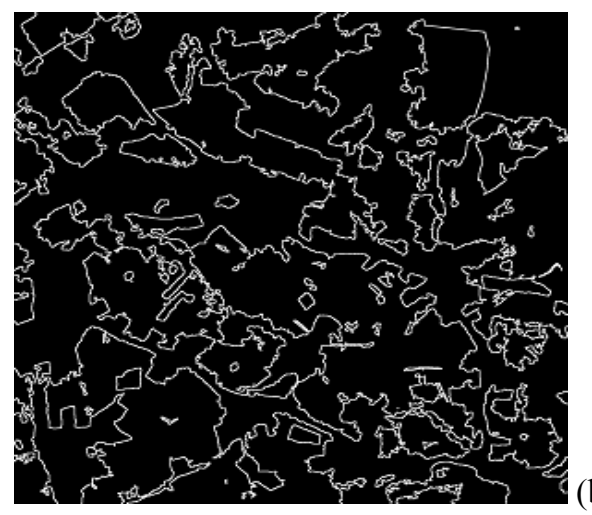

(b)

Fig 5. (a) Classified image (b) The boundary tracing of the classified objects

Obviously, the proposed method could distinguish between tea gardens and rest of non-vegetation areas with high accuracy. However, there were some difficulties in distinguishing tea gardens from other types of vegetation (forest, other types of trees) whose spectral signatures are similar to that of tea gardens. To deal with this situation, a manual editing step was also included with the software so that users can edit the miss classified objects.

\section{Experimental Results}

In this study, spectral signatures were employed for extraction of tea gardens from 1:5000 scaled orthophoto maps. Four bands (red, green, blue, and near infrared) were used for the tea garden classification. Although various sophisticated methods have been proposed for evaluation e.g. (Whiteside, Maier, and Boggs 2014; Zhang 2001; Zylshal et al. 2016), yet we employed most commonly used area based accuracy assessment. Both classified and manually digitized images were first converted into binary images, with white color representing tea gardens while black color indicating background and then compared to find the accuracy of the propose method. We used the area-based method for accuracy assessment proposed by (Whiteside, Maier, and Boggs 2014).

The experiments were performed on 10 images to evaluate the proposed method. Table 2 summarized the performance of the propose technique. The overall accuracy obtained was $89 \%$, user accuracy $88 \%$, producer accuracy $93 \%$, false positive were $12 \%$ and false negatives were $7 \%$. Each obtained tea gardens from proposed method were compared with manually delineated tea gardens. To obtain overall accuracy, we summed up area of intersection for all images and divided it by total area of the ground truth images.

\begin{tabular}{|l|c|c|c|c|c|}
\hline Class & OA(\%) & UA(\%) & PA(\%) & $\begin{array}{c}\text { FP } \\
(\%)\end{array}$ & FN(\%) \\
\hline $\begin{array}{l}\text { Tea } \\
\text { Gardens }\end{array}$ & 89 & 88 & 93 & 12 & 7 \\
\hline
\end{tabular}

Table 2. Area-based accuracy assessment

Suppose, C represents the area of classified image obtained from proposed method and $\mathrm{R}$ be the area of manually digitized images (ground truth). $\mathrm{C} \cap \mathrm{R}$ is the area of intersection between $\mathrm{C}$ and $\mathrm{R}, \mathrm{C} \cap \neg \mathrm{R}$ represents the false positive (FP), $\neg \mathrm{C} \cap \mathrm{R}$ represent false negatives (FN). Moreover, the producer's accuracy (PA) and user's accuracy (UA) were also calculated to assess the classification accuracy for each class. Finally, the overall accuracy (OA) was obtained by summing up the area of intersection for all images and dividing by the all ground truth area. The summary of the assessment measures are summarized in Table 3.

\begin{tabular}{|l|c|}
\hline \multicolumn{1}{|c|}{ Measure } & Formula \\
\hline User's Accuracy & $\frac{|C \cap R|}{|C|}$ \\
\hline Producer's Accuracy & $\frac{|C \cap R|}{|R|}$ \\
\hline False Positives & $\frac{|C \cap \neg R|}{|R|}$ \\
\hline False Negatives & $\frac{|\neg C \cap R|}{|R|}$ \\
\hline
\end{tabular}

Table 3. Area-based accuracy assessment

In Fig. 5 the classification results are presented. It can be noticed that the proposed method was effective in identifying the tea gardens from 1:5000 scaled orthophoto maps. Fig. 5 (a) is the original image with 4 bands (R,G,B,NIR), (b) is the classified binary image, where white color represent tea gardens while black indicated rest of classes. In (c) binary image is overlaid on the original image to see the mapping clearly. For (d) the contours of each object in (b) is calculated and over laid on the original image for visual interpretations.
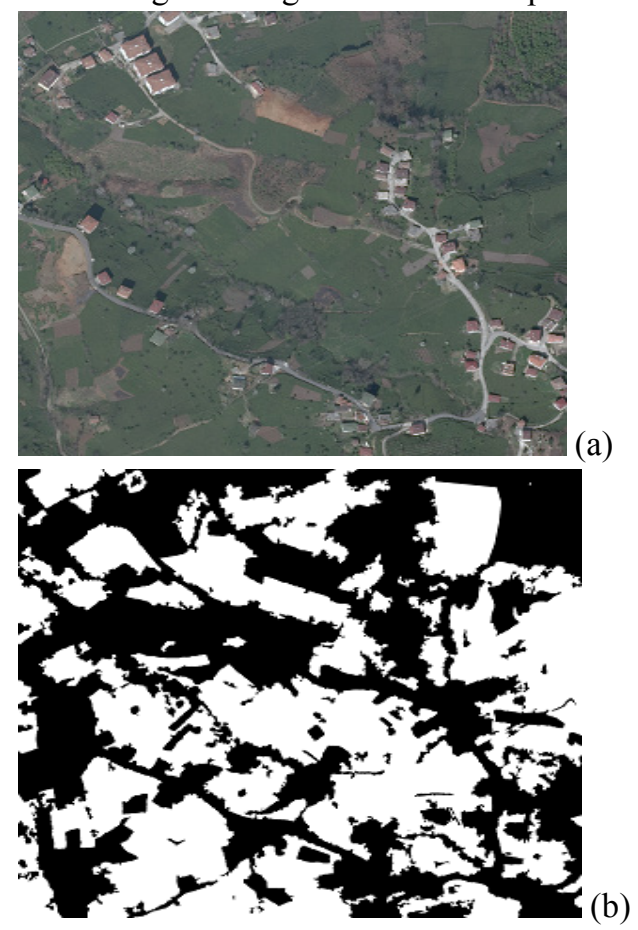

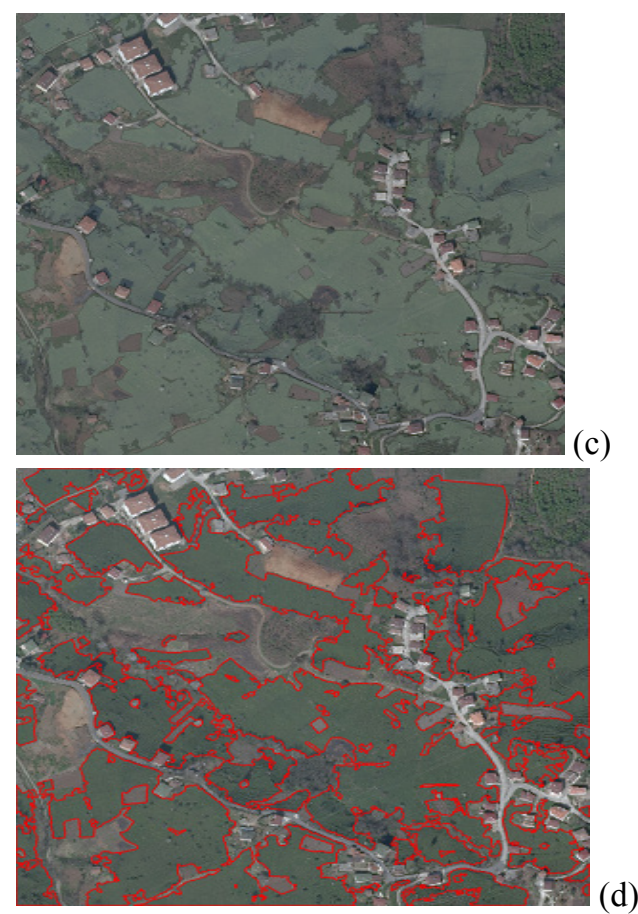

Fig. 5 (a) Original digital orthophotomap (4 bands), (b) Binary image with white pixels showing tea gardens, (c) binary image overplayed on original image and (d) Contours drawn around tea gardens

The proposed methodology was implemented using MATLAB and Emgu CV. The experiments were run on a machine having RAM $8 \mathrm{GHz}$ CPU. The computational complexity of the proposed method for various sizes of images is shown in Fig 6. It is observed that most of the processing time was consumed for image segmentation and classification tasks. The run time complexity for both algorithms is approximately $\mathrm{O}(\mathrm{N} 2)$. The algorithm can be accelerated by employing parallel programming techniques and GPU enabled processing.

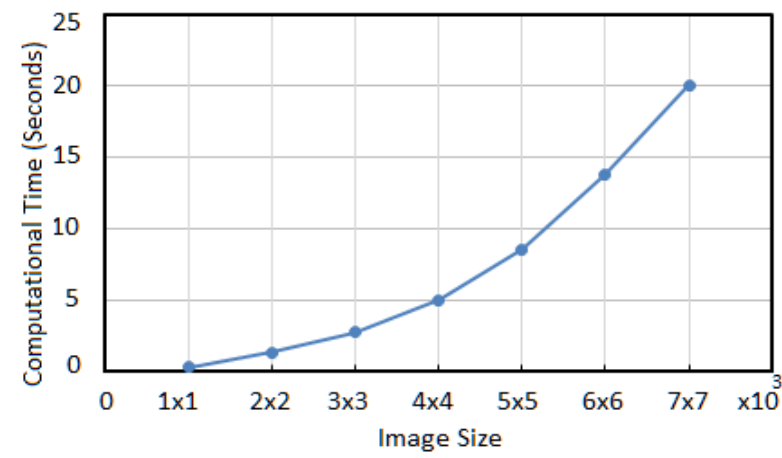

Fig. 6 Computational complexity of the proposed system. Plot of processing time against size of the input image.

\section{Conclusions}

Photogrammetry and remote sensing data is very important source of information that is used for tree species identification over large geographical areas. We showed the usefulness of spectral signatures for tea garden extraction from 1:5000 scaled digital orthophoto maps. NDVI was first derived from the orthophoto maps to distinguish between vegetation and non-vegetation areas. The raw pixels were then grouped into an object representation by exploiting both spatial and textural features. Then, geometrical constraints and morphological processing was performed to reduce the noisy elements. Finally, a spectral signature based range filter was applied to extract tea gardens. The method presented here for tea garden extraction can also be adopted for other types of vegetation identification by only altering the model parameters.

In future work, we plan to extend our method for land cover classification (water bodies, bare land, build areas) by including other type of vegetation indexes such as greenness, extended greenness index etc. Additionally, inclusion of a machine learning approach may be useful for classification stage. This may require derivation of new features from segmented objects and apply a feature selection approach to identify robust feature set that could be employed for classifying objects into respective classes.

\section{Acknowledgements}

This study is a part of TEYDEP Project entitled "Development of Object Based Neural Network Image Pro-cessing System Determination of Vegetation and Forestry Boundaries" (Project Nr. 7140512). It was supervised by EMI Group-Turkey, and consulted by Prof. Dr. Bulent Bayram.

\section{References}

Adhikari, H., J. Heiskanen, E. E Maeda, and P. K. E. Pellikka. 2015. "Does Topographic Normalization of Landsat Images Improve Fractional Tree Cover Mapping in Tropical Mountains?" In International Archives of the Photogrammetry, Remote Sensing and Spatial Information Sciences, , 261-67. http://www.intarch-photogramm-remote-sens-spatial-inf-sci.net/XL-7W3/261/2015/.

Alvarez, F, T Catanzarite, J R Rodríguez-Pérez, and D Nafría. 2010. "Radiometric Calibration and Evaluation of UltraCamX and Xp Using Portable Reflectance Targets and Spectrometer Data. Application to Extract Thematic Data from Imagery Gathered by the National Plan of Aerial Orthophotography (PNOA)." International Calibration and Orientation Workshop EuroCOW.

Bandyopadhyay, Madhurima, Jan a. N. van Aardt, and Kerry Cawse-Nicholson. 2013. "Classification and Extraction of Trees and Buildings from Urban Scenes Using Discrete Return LiDAR and Aerial Color Imagery." In Spie, ， 873105-873105-9. http://proceedings.spiedigitallibrary.org/proceeding.asp $\mathrm{x}$ ?doi=10.1117/12.2015890.

Blaschke, T. 2010. "Object Based Image Analysis for Remote Sensing." ISPRS Journal of Photogrammetry and Remote Sensing 65(1): 2-16. http://linkinghub.elsevier.com/retrieve/pii/S0924271609 000884 (July 10, 2014).

Colgan, Matthew S., Claire A. Baldeck, Jean baptiste Féret, and Gregory P. Asner. 2012. "Mapping Savanna 
Tree Species at Ecosystem Scales Using Support Vector Machine Classification and BRDF Correction on Airborne Hyperspectral and LiDAR Data." Remote Sensing 4(11): 3462-80.

Dalponte, Michele et al. 2013. "Tree Species Classification in Boreal Forests with Hyperspectral Data." IEEE Transactions on Geoscience and Remote Sensing 51(5): 2632-45.

Deng, Songqiu et al. 2014. "Interpretation of Forest Resources at the Individual Tree Level at Purple Mountain, Nanjing City, China, Using WorldView-2 Imagery by Combining GPS, RS and GIS Technologies." Remote Sensing: 87-110.

Gu, Yanfeng et al. 2016. "Nonlinear Multiple Kernel Learning with Multiple-Structure-Element Extended Morphological Profiles for Hyperspectral Image Classification." IEEE Transactions on Geoscience and Remote Sensing 54(6): 3235-47.

Jeevalakshmi, D, S Narayana Reddy, and B Manikiam. 2016. "Land Cover Classification Based on NDVI Using LANDSAT8 Time Series : A Case Study Tirupati Region." 560056: 1332-35.

Li, Xiuxia et al. 2015. "A NEW REGION GROWINGBASED SEGMENTATION METHOD FOR HIGH RESOLUTION REMOTE SENSING IMAGERY." In IEEE International Geoscience and Remote Sensing Symposium, , 4328-31.

Palenichka, Roman, Frederik Doyon, Ahmed Lakhssassi, and Marek B. Zaremba. 2013. "Multi-Scale Segmentation of Forest Areas and Tree Detection in Lidar Images by the Attentive Vision Method." IEEE Journal of Selected Topics in Applied Earth Observations and Remote Sensing 6(3): 1313-23.

Paula., Pedro Luiz de, Luiz S. Oliveira, Alceu de Souza Britto Jr., and Robert Sabourin. 2010. "Forest Species Recognition Using Color-Based Features." In International Conference on Pattern Recognition, , 4178-81..

Pena-Barragana, J.M et al. 2004. "Assessing Land-Use in Olive Groves from Aerial Photographs." Agriculture, Ecosystems and Environment 103(1): 117-22.

Pham, Lien T.H., Lars Brabyn, and Salman Ashraf. 2016. "Combining QuickBird, LiDAR, and GIS Topography Indices to Identify a Single Native Tree Species in a Complex Landscape Using an Object-Based Classification Approach." International Journal of Applied Earth Observation and Geoinformation 50: 187-97.

http://linkinghub.elsevier.com/retrieve/pii/S0303243416 300460.

Qin, Rongjun. 2014. “An Object-Based Hierarchical Method for Change Detection Using Unmanned Aerial Vehicle Images.” Remote Sensing 6(9): 7911-32.

Sugumaran, R., and M. Voss. 2007. "Object-Oriented Classification of LIDAR-Fused Hyperspectral Imagery for Tree Species Identificationin an Urban Environment.” In Urban Remote Sensing Joint Event,.
Tzotsos, A, and D Argialas. 2008. "Support Vector Machine Classification for Object- Based Image Analysis.” In Analysis, , 663-77.

Walter, Volker. 2004. "Object-Based Classification of Remote Sensing Data for Change Detection." ISPRS Journal of Photogrammetry and Remote Sensing 58(34): $225-38$.

Whiteside, Timothy G., Stefan W. Maier, and Guy S. Boggs. 2014. "Area-Based and Location-Based Validation of Classified Image Objects.” International Journal of Applied Earth Observation and Geoinformation 28(1): 117-30. http://dx.doi.org/10.1016/j.jag.2013.11.009.

Zhang, Yun. 2001. “A Spectral and Spatial Information Integrated Approach for Tree Extraction from HighResolution Digital Imagery." In Second Digital Earth Conference, , 1-10.

Zhou, Lihang et al. 2016. "Monitoring the Atmospheric Environment with Joint Polar Satellite System (JPSS) Remote Sensing Data Products." In IEEE International Geoscience and Remote Sensing Symposium (IGARSS), IEEE, 3945-48. http://ieeexplore.ieee.org/document/7730025/ (January 31, 2017).

Zylshal et al. 2016. "A Support Vector Machine Object Based Image Analysis Approach on Urban Green Space Extraction Using Pleiades-1A Imagery." Modeling Earth Systems and Environment 2(54): 54. http://link.springer.com/10.1007/s40808-016-0108-8. 